\title{
SOLAR PROMINENCES EMBEDDED IN FLUX ROPES: MORPHOLOGICAL FEATURES AND DYNAMICS FROM 3D MHD SIMULATIONS
}

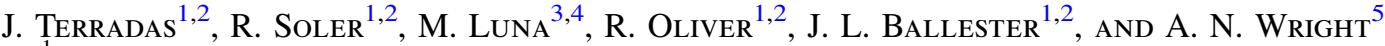 \\ ${ }^{1}$ Departament de Física, Universitat de les Illes Balears, E-07122 Palma de Mallorca, Spain; jaume.terradas@uib.es \\ ${ }^{2}$ Institute of Applied Computing \& Community Code (IAC), UIB, Spain \\ ${ }^{3}$ Instituto de Astrofsíca de Canarias, E-38205 La Laguna, Tenerife, Spain \\ ${ }_{5}^{4}$ Departamento de Astrofísica, Universidad de la Laguna, E-38205 La Laguna, Tenerife, Spain \\ ${ }^{5}$ School of Mathematics and Statistics, University of St Andrews, St Andrews, KY16 9SS, UK \\ Received 2015 December 2; accepted 2016 February 22; published 2016 March 30
}

\begin{abstract}
The temporal evolution of a solar prominence inserted in a three-dimensional magnetic flux rope is investigated numerically. Using the model of Titov \& Démoulin under the regime of weak twist, the cold and dense prominence counteracts gravity by modifying the initially force-free magnetic configuration. In some cases a quasi-stationary situation is achieved after the relaxation phase, characterized by the excitation of standing vertical oscillations. These oscillations show a strong attenuation with time produced by the mechanism of continuum damping due to the inhomogeneous transition between the prominence and solar corona. The characteristic period of the vertical oscillations does not depend strongly on the twist of the flux rope. Nonlinearity is responsible for triggering the Kelvin-Helmholtz instability associated with the vertical oscillations and that eventually produces horizontal structures. Contrary to other configurations in which the longitudinal axis of the prominence is permeated by a perpendicular magnetic field, like in unsheared arcades, the orientation of the prominence along the flux rope axis prevents the development of Rayleigh-Taylor instabilities and therefore the appearance of vertical structuring along this axis.
\end{abstract}

Key words: magnetic fields - plasmas - Sun: corona

Supporting material: animations

\section{INTRODUCTION}

Active region prominences are often associated with magnetic structures that seem to have a flux rope geometry, i.e., a coherent structure in which magnetic field lines wind around a central axis. Several attempts have been made to provide the theoretical background, based on the equations of magnetohydrostatics that describe this kind of magnetic configuration (e.g., Kuperus \& Raadu 1974; Priest et al. 1989; Low \& Zhang 2004; Blokland \& Keppens 2011; Hillier \& van Ballegooijen 2013). In most cases the heavy prominence is absent from the magnetic configuration (see the review of Mackay et al. 2010), and/or the magnetic field lines do not connect to the photosphere since the models are mostly $2 \mathrm{D}$. These models are quite limited and restrain a proper understanding of the dynamics of suspended prominences. Only recently, it has been possible to create a self-consistent plasmacarrying flux rope (Xia et al. 2014b) and to produce an in situ condensation to a prominence due to thermal instability (Xia et al. 2014a).

Nowadays it is clear that solar prominences are very dynamic, and several observed phenomena have been identified as the result of the development of different plasma instabilities, such as the magnetic Rayleigh-Taylor (MRT) instabilities or the Kelvin-Helmholtz instabilities (KHIs) (e.g., Ryutova et al. 2010). Other dynamic events affecting the whole prominence body are the ubiquitous large-amplitude oscillations associated with winking filaments (see the review of Tripathi et al. 2009) or the longitudinal oscillations (Li \& Zhang 2012; Luna et al. 2014). It is evident that an accurate theoretical description of these oscillations, usually done in terms of magnetohydrodynamic (MHD) waves, demands realistic prominence models, and the incorporation of the three-dimensional (3D) geometry is essential.

Solar prominences are in general quite inhomogeneous, and this property has important consequences regarding oscillations, which are susceptible to experiencing resonant damping. Here we understand by resonant absorption or resonant damping the process in which the energy of the global oscillation is transferred to nonhomogeneous layers. Since in our problem the oscillations are not externally driven and monochromatic, the energy is not transferred to a single position (the resonant position) in the layer. Instead, the energy accumulates around the resonant position, i.e., where there is a perfect match between the real part of the frequency of the global mode, often called a quasi-mode, and the frequency of the local Alfvén modes. Importantly, there is also energy transferred to frequencies around the resonant Alfvén frequency. For this reason, we think that the term resonant absorption in the case of the initial value problem is indeed not very accurate since strictly speaking the energy is not transferred to a single resonant position. A more general name for this mechanism of attenuation is continuum damping (e.g., Sedláček 1995). Another way to refer to this process is mode conversion or mode coupling to natural Alfvén oscillations (e.g., Rae 1982; Lee \& Roberts 1986; Pascoe et al. 2010; Hood et al. 2013; Ruderman \& Terradas 2013). The term phase mixing has also been used in the literature (e.g., Grossmann \& Tataronis 1973; Tataronis \& Grossmann 1973), while in magnetospheric plasmas the term field line resonance (FLR) is usually preferred.

An attractive alternative to the classical interpretation of the quasi-mode is based on the proper superposition of Alfvén continuum modes (Cally 1991; Mann et al. 1995). Recently, 
Soler \& Terradas (2015) have investigated the generation of small scales in nonuniform solar magnetic flux tubes. Using a modal expansion, the initial global MHD transverse displacement has been expressed as a superposition of Alfvén continuum modes that are phase-mixed as time evolves. The comparison with the results of the quasi-mode indicates that the modal analysis is more intelligible from the physical point of view since it describes both the damping of global transverse motions and the building up of small scales due to phase mixing. It is not surprising that the term phase mixing has also been used in the past to refer to the conversion of energy from large to small scales (see Tataronis \& Grossmann 1973).

In prominences the strongest density inhomogeneity is usually associated with the layers that connect the prominence core with the corona through the prominence-corona transition region (PCTR). Therefore, the inhomogeneous PCTR is crucial for the existence of Alfvén continuum modes that are eventually responsible for the damping. Recently, the idea behind resonant damping has been applied to prominences/ threads (Arregui et al. 2008, 2011; Soler et al. 2009, 2010a; Arregui \& Ballester 2011; Antolin et al. 2015; Okamoto et al. 2015). The continuum damping is not new and has been investigated in the past under different frameworks: laboratory plasmas (Tataronis \& Grossmann 1973; Chen \& Hasegawa 1974; Poedts et al. 1992), coronal loop oscillations (e.g., Ionson 1978; Hollweg 1987; Hollweg \& Yang 1988; Sakurai et al. 1991; Goossens et al. 1992, 2002; Ruderman \& Roberts 2002), and magnetospheric plasmas (Southwood 1974; Mann et al. 1995; Wright \& Rickard 1995). In the previous studies both the driven and the initial value problems have been addressed, and the term resonant absorption has been used in most of the cases except for magnetospheric plasmas. For historical reasons we tend to keep using the name resonant absorption.

With the goal of improving the existing magnetohydrostatic prominence models, Terradas et al. (2015) have studied the morphology of a prominence suspended in a 3D arcade configuration. In that work, the authors have found that the prominence is especially prone to developing MRT instabilities (see also Hillier et al. 2012a, 2012b). In the present study we extend the work of these authors to a twisted flux rope, more representative of active region prominences than quiescent prominences. In particular, we choose the magnetic configuration constructed by Titov \& Démoulin (1999). This 3D magnetic model can represent a wide variety of flux ropes, depending on the parameters, and has the advantage that it is easily implemented using analytic expressions for the 3D magnetic field. The aim of this paper is to understand the main morphological features of a prominence embedded in a flux rope for different values of the magnetic twist. The models studied here are global (the fine structures of the prominence, i.e., threads, are not resolved) and could be used, in future studies, to analyze large-amplitude oscillations associated with winking filaments or with longitudinal oscillations.

This study is challenging from several points of view. First, the process of resonant absorption, associated with the oscillations, has the particularity that decreasing spatial scales are continuously built with time through phase mixing (e.g., Heyvaerts \& Priest 1983). This inevitably leads to a situation in which the grid resolution is unable to capture the small spatial scales, and this produces an artificial (numerical) dissipation of energy in the system. We have conceived specific numerical experiments to understand the effect of numerical dissipation on the results. The process of wave leakage, i.e., the emission of fast magnetoacoustic waves from the prominence, can also operate in our model and produce a physical energy loss from the system. We have also devised particular simulations, based on the application of special boundary conditions, to investigate this problem. Second, and closely related to the first point, is the presence of instabilities. In particular, for the configuration studied here KHIs due to the strong shear velocities at the prominence edges play a relevant role in the morphology of the structure. Eddies are generated at the PCTR and energy cascades to small spatial scales until numerical dissipation becomes important. Another complication in this study is that the numerical techniques applied to capture the vigorous motions associated with the KHI may impede a proper analysis of possible stationary states of the system. This issue is properly treated in this work by the use of specific numerical techniques. Thus, the problem investigated here involves several ideal mechanisms that are not independent one from another and generate a rich dynamism in the system, but at the same time we intend to find out potential stationary regimes.

\section{INITIAL SETUP}

In our model the background configuration is a vertically stratified atmosphere due to constant gravity. Background density and gas pressure are exponentially decreasing with height, and from the ideal gas law temperature is uniform (isothermal background) and chosen to be $1 \mathrm{MK}$, which is representative of coronal conditions. This atmosphere is permeated by a magnetic field following the Titov \& Démoulin (1999) model, which is a 3D toroidal force-free model that contains a poloidal component of the magnetic field. In the construction of the model it is assumed that the small radius of the flux rope is much smaller than the large radius of the toroid. This model has been used in the past mainly to study eruptions and coronal mass ejections (CMEs; see Török \& Kliem 2003; Török et al. 2004). Here we concentrate on a stable configuration with respect to the kink instability that appears when the twist is above a given threshold. The amount of twist is controlled by the parameter $N_{t}$. Values of 8.5 (strong), 4.3 (moderate), and 2.2 (weak twist) are considered in this work. It is worth mentioning that for the strongest twist, the ratio between the toroidal and azimuthal magnetic field component is around 2 at the edge of the flux rope, but the field lines do not have more than three turns, in agreement with observations of stable flux ropes. Examples of the three magnetic configurations are found in Figure 1. Note that for $N_{t}=4.3$ and 2.2 the configurations resemble arcades whose magnetic shear increases with height. In the three models the small radius of the toroidal flux rope is $a=10 \mathrm{Mm}$, while the big radius is $R=140 \mathrm{Mm}$. The height of the center of the flux rope is $20 \mathrm{Mm}$.

The prominence is represented by a 3D density enhancement with respect to the background density and is located inside the flux rope. A simple Gaussian profile in each direction is used. The characteristic dimensions of the prominence are width of $5 \mathrm{Mm}$, height of $10 \mathrm{Mm}$, and a length of $20 \mathrm{Mm}$. The center of the prominence is initially situated at $15 \mathrm{Mm}$ from the base of the corona. This density enhancement is also represented in Figure 1 in a $3 \mathrm{D}$ view pointing along the longitudinal axis of 

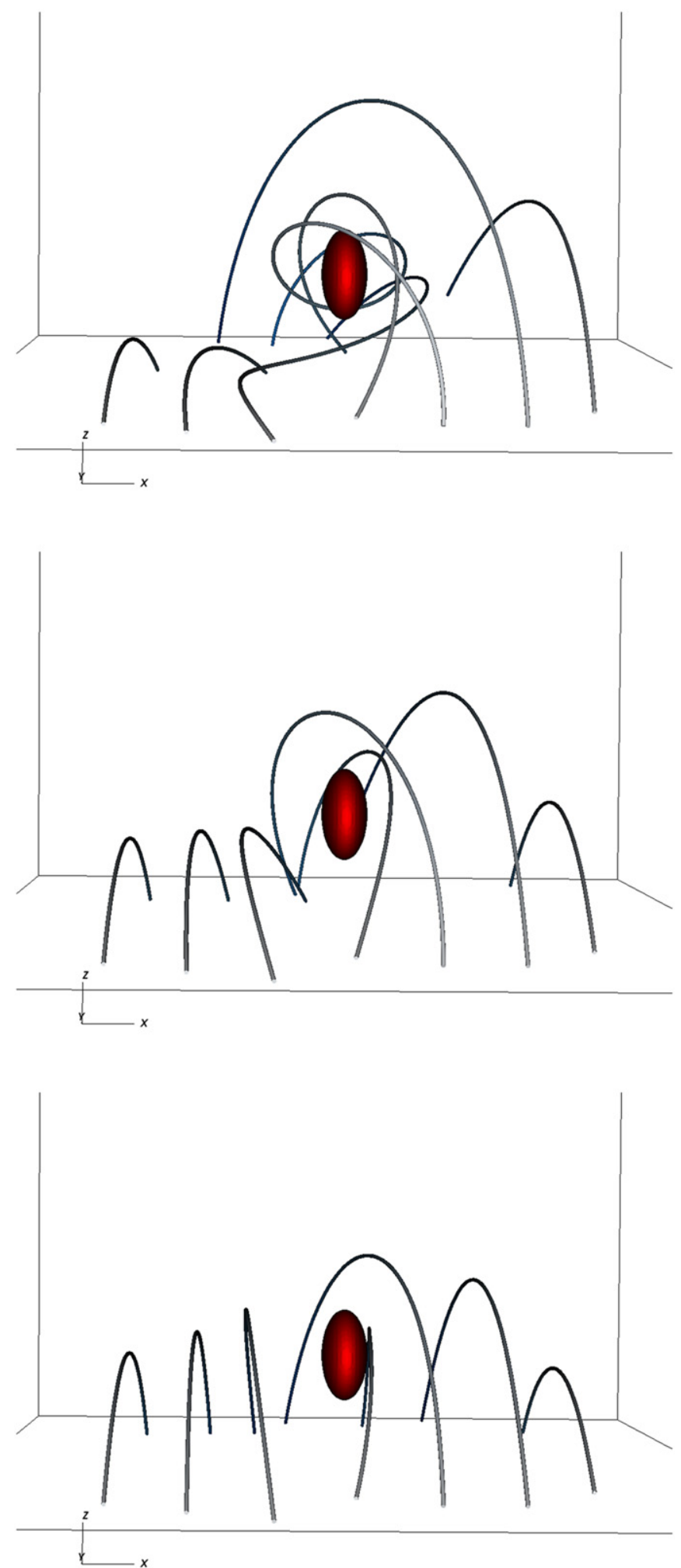

Figure 1. Magnetic field configuration and initial density enhancement (in red colors) for $N_{t}=8.5$ (top panel), $N_{t}=4.3$ (middle panel), and $N_{t}=2.2$ (bottom panel). The same magnetic field lines rooted at the base of the corona have been selected in the three plots.

the prominence, i.e., along the $y$-direction. Different values for the density enhancement, and therefore total masses of the prominence, have been considered, allowing us to study situations of extremely light prominences to cases with total masses similar to the ones inferred from observations. In particular, the maximum density enhancement used in this work is 30 times the coronal density. Although this value is a bit low in comparison with the densities estimated from observations, it gives a total mass of $1.8 \times 10^{10} \mathrm{~kg}$, which is typical of light prominences. Larger density enhancements, and therefore total masses, can lead to a situation in which the magnetic structure is unable to sustain the prominence (see Terradas et al. 2015). Increasing the magnetic field provides additional magnetic support to the prominence since the magnetic force scales as $B^{2}$. For example, if we double the magnetic field, the Lorentz force is four times larger, and therefore a prominence four times more massive could be sustained by this new field. The intensity of the magnetic field determines, together with the density, the value of the Alfvén speed. In the solar corona we take as a reference value $1.8 \times 10^{3} \mathrm{~km} \mathrm{~s}^{-1}$ (this is consistent with the estimations of Verwichte et al. [2013], based on seismological, magnetic extrapolation and spectral methods), meaning that the magnetic field is constrained by this velocity. In our simulations the maximum value of $B_{z}$ at the base of the corona is $5 \mathrm{G}$, and it fulfills the restriction given by the typical coronal Alfvén speed. With this magnetic field and the corresponding gas pressure of a plasma at $1 \mathrm{MK}$, the plasma- $\beta$ is in the range $0.02-0.09$. We are clearly in a low- $\beta$ regime, and this guides the dynamic processes that take place in the configuration. Stronger magnetic fields, which are feasible because we are considering an active region prominence, would augment too much the value of the Alfvén speed and decrease the already low value of the plasma- $\beta$.

Regarding the geometry of the magnetic field, it is worth mentioning that the magnetic support of the prominence when the longitudinal axis is essentially along the magnetic field, such as in a flux rope with low twist like the one in the bottom panel of Figure 1, is less efficient than a situation in which the magnetic field is perpendicular to this axis. This would correspond, in our configuration, to a prominence with the longitudinal axis pointing in the $x$-direction. The reason is that in this case the prominence is permeated by more magnetic field, meaning that the total magnetic force is stronger. This simple geometrical feature of our model has important consequences with respect to the total amount of mass that can be sustained by our configuration and, as we show later, regarding the MRT instability. In situations with stronger magnetic twist, as in the top panel of Figure 1, the magnetic support increases owing to the presence of local magnetic dips, but since we do not consider more than three turns of field lines, this additional support is not significantly enhanced in our configuration.

\section{METHOD AND BOUNDARY CONDITIONS}

The background and the magnetic field are initially in equilibrium. Given an initial localized density distribution representing the prominence, which is not in equilibrium with the environment, the system is allowed to evolve with time. To investigate the dynamics of our configuration, the ideal 3D MHD equations are numerically solved. The equations are the following:

$$
\frac{\partial \rho}{\partial t}+\nabla \cdot(\rho \boldsymbol{v})=0
$$




$$
\begin{gathered}
\frac{\partial \rho \boldsymbol{v}}{\partial t}+\nabla \cdot\left(\rho \boldsymbol{v} \boldsymbol{v}+p \boldsymbol{I}-\frac{\boldsymbol{B B}}{\mu}+\frac{\boldsymbol{B}^{2}}{2 \mu}\right)=\boldsymbol{\rho} g, \\
\frac{\partial \boldsymbol{B}}{\partial t}=\nabla \times(\boldsymbol{v} \times \boldsymbol{B}), \\
\frac{\partial p}{\partial t}+\nabla \cdot(\gamma p \boldsymbol{v})=(\gamma-1) \boldsymbol{v} \cdot \nabla p,
\end{gathered}
$$

where $\boldsymbol{I}$ is the unit tensor, $\boldsymbol{g}$ is the gravitational acceleration, and the rest of the symbols have their usual meaning. Temperature does not appear explicitly in the previous equations, but it is easily calculated using the ideal gas law and the values of pressure and density. The nonlinear MHD equations are solved in a Cartesian coordinate system. The gravity force points in the negative $z$-direction, and the longitudinal axis of the prominence is along the $y$-direction (see Figure 1). Our model does not include a density transition between the corona and the photosphere, and the plane at $z=0$ represents the base of the corona. The dimensions of the domain are $-50 \mathrm{Mm}<x<50 \mathrm{Mm},-100 \mathrm{Mm}<y<100 \mathrm{Mm}$, and $0<z<60 \mathrm{Mm}$. Different numerical resolutions have been considered, and the best resolution achieved with the present computational resources is $300 \mathrm{~km}$.

The previous equations are solved using the code MoLMHD, which has been updated to be efficient in achieving stationary solutions but at the same time is robust in the treatment of weak shocks. With this goal in mind, the order of the spatial derivatives has been raised to six, and the scheme has been combined with a fifth-order WENO method. The rest of the details about the code can be found in Terradas et al. (2015) and references therein.

Line-tying boundary conditions are applied at the base of the corona, meaning that the three components of the velocity, $v$, are set to zero, the magnetic component perpendicular to the boundary is kept constant, $B_{z}$ in our case, and the rest of the variables have their spatial derivatives equal to zero. For the rest of the boundaries in the computational box, i.e., lateral and top planes, two types of conditions are investigated: perfectly reflecting boundaries, i.e., line-tying (this condition is referred to as closed boundary conditions), and nonreflecting boundaries. This last condition is implemented through the method of the perfectly matched layer (PML). The method is based on the work of Berenger (1994) and Hu (2001) (see also Parchevsky \& Kosovichev 2007), and it is efficient in absorbing outgoing waves. Hereafter, these boundary conditions are referred to as open boundary conditions.

\section{TEST CASE}

We start with the configuration explained in Section 2, but we do not introduce at this stage a prominence inside the flux rope. This allows us to verify that we have correctly implemented the force-free Titov and Demoulin model since the structure should be close to a static equilibrium. The flux rope with the strongest twist $\left(N_{t}=8.5\right)$, and therefore the strongest current, is chosen. Closed boundaries, i.e., line-tying conditions at all the sides of the computational box, are imposed.

In the top panel of Figure 2some magnetic field lines are represented, together with the toroidal component of the magnetic field $\left(B_{y}\right)$ at the central plane of the configuration. The results of the evolution, not shown here, indicate that the
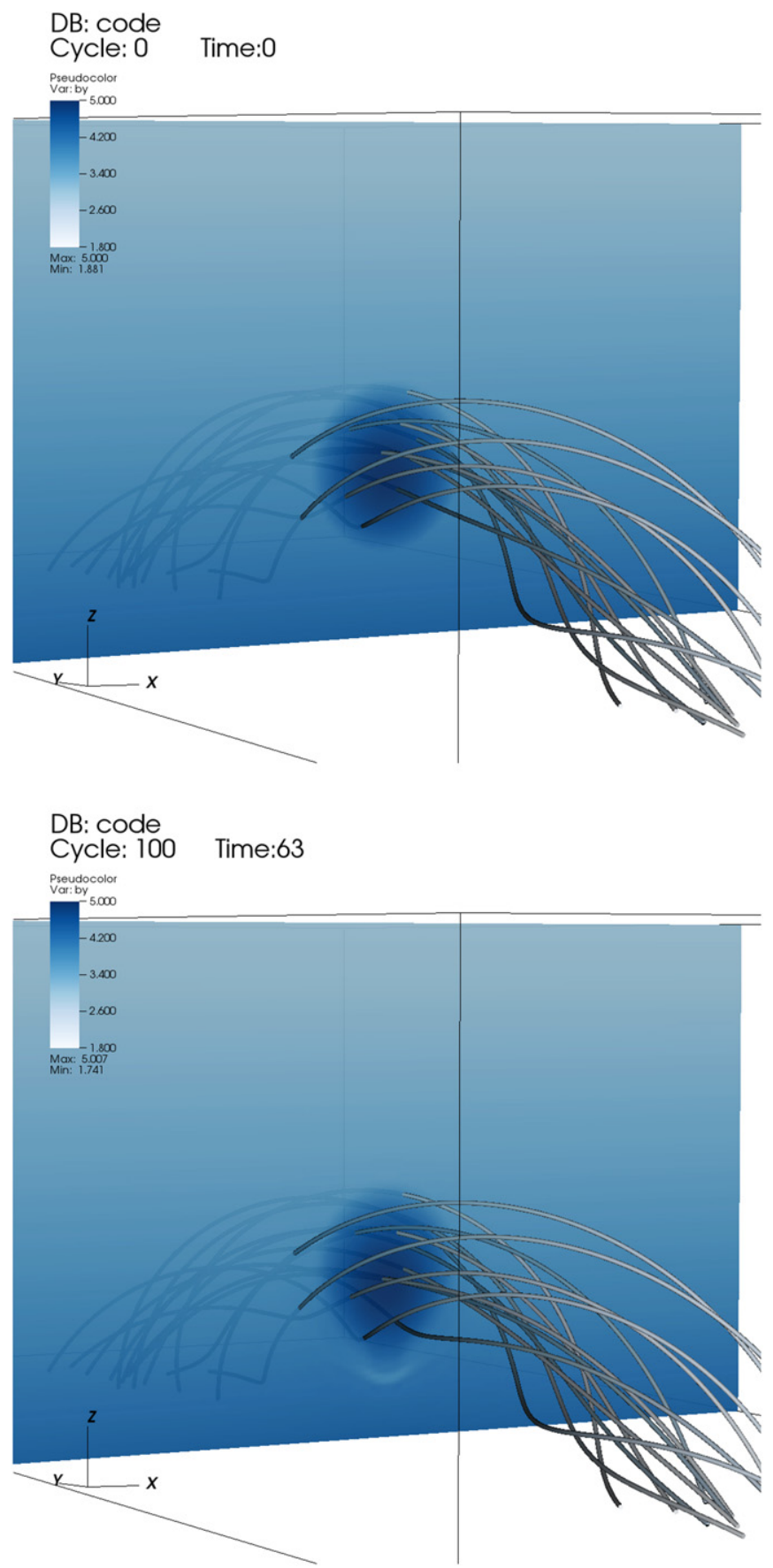

Figure 2. Snapshot of the flux rope configuration for $N_{t}=8.5$ (strong twist). The $B_{y}$ component of the magnetic field is plotted at the central plane together with some specific magnetic field lines. The top panel corresponds to the test case without the prominence enhancement, while in the bottom panel the dense prominence inside the flux rope is present (but not visible in this plot).

system is in essentially the same state as in the beginning of the simulation, meaning that the magnetic field model is indeed force-free. We find rather small flows, of the order of $2 \mathrm{~km} \mathrm{~s}^{-1}$, associated with the relaxation process of the magnetic configuration, which are eventually attenuated. The flux rope cross section keeps the initial circular shape, indicating that the thin-tube approximation used to construct the toroidal model works well $(a / R=0.071$ in our model). Evidences of kink or helical instabilities, which eventually would destroy the equilibrium, are not present in the simulation. This is the 
expected behavior since we have intentionally imposed a small number of turns of the magnetic field lines around the flux rope axis.

The presence of the flux rope in the global arcade configuration produces an increase in the local Alfvén speed $\left(v_{A}=B / \sqrt{\mu_{0} \rho}\right)$. It is known that to have an efficient waveguide that is able to trap energy, a minimum in the Alfvén speed is required. This means that without a dense prominence inside the flux rope, which will produce an important decrease of the Alfvén speed, the toroidal magnetic structure with just the background density is unable to act as a wave guide, at least for fast MHD transverse waves.

\section{FLUX ROPE WITH AN EMBEDDED PROMINENCE}

\subsection{Global Features}

The situation is different when a heavy prominence is incorporated into the flux rope. The bottom panel of Figure 2 shows the results after $1 \mathrm{hr}$ of evolution, and several differences with respect to the simulation described in Section 4 are evident (compare with the top panel of Figure 2). The cross section of the flux rope is no longer purely circular and shows an oval shape. The prominence is located initially at $z_{0}=15 \mathrm{Mm}$, and because of the gravity force, the magnetic structure and the prominence are pushed downward. The results of this simulation show that the upper part of the flux rope is essentially unaltered while the lower part, where the prominence was introduced, suffers the strongest change in the toroidal magnetic field. A close inspection of bottom panel of Figure 2 reveals that $B_{y}$ underneath the prominence shows a significant decrease. Note also that from this figure the magnetic field lines inside the flux rope are slightly different with respect to the top panel of Figure 2 because of the presence of the prominence.

A density isocontour and some magnetic field lines are represented in Figure 3 at three different times. The shape of the prominence changes from the initial Gaussian profile to a structure that is more irregular and elongated. In this simulation the prominence is suspended above the base of the corona mainly owing to the magnetic restoring forces associated with the dips introduced by twist that counteract gravity. The mass tends to be aligned with the flux rope axis.

In our model the temperature at the core of the prominence is typically around $2.6 \times 10^{4} \mathrm{~K}$ and connects smoothly with the much hotter coronal environment, at $1 \times 10^{6} \mathrm{~K}$. This low temperature at the prominence core is essentially determined by the initial density enhancement. We have found that gas pressure is basically constant across the prominence, meaning that an increase in density leads to a decrease in temperature according to the ideal gas law. The connection of the core of the prominence with the external corona is through the PCTR. The size of this transition layer is mostly determined by the initial shape of the density enhancement, which is Gaussian in our case.

We have repeated the simulation for the configurations with intermediate and weak twist, and the results for the three twisted configurations at the same instant (63.6 minutes) are shown in Figure 4. For the three configurations we obtain suspended prominences, and this is not surprising for the cases with strong and moderate twist since the magnetic structure has dips. Nevertheless, even in the situation with weak twist we

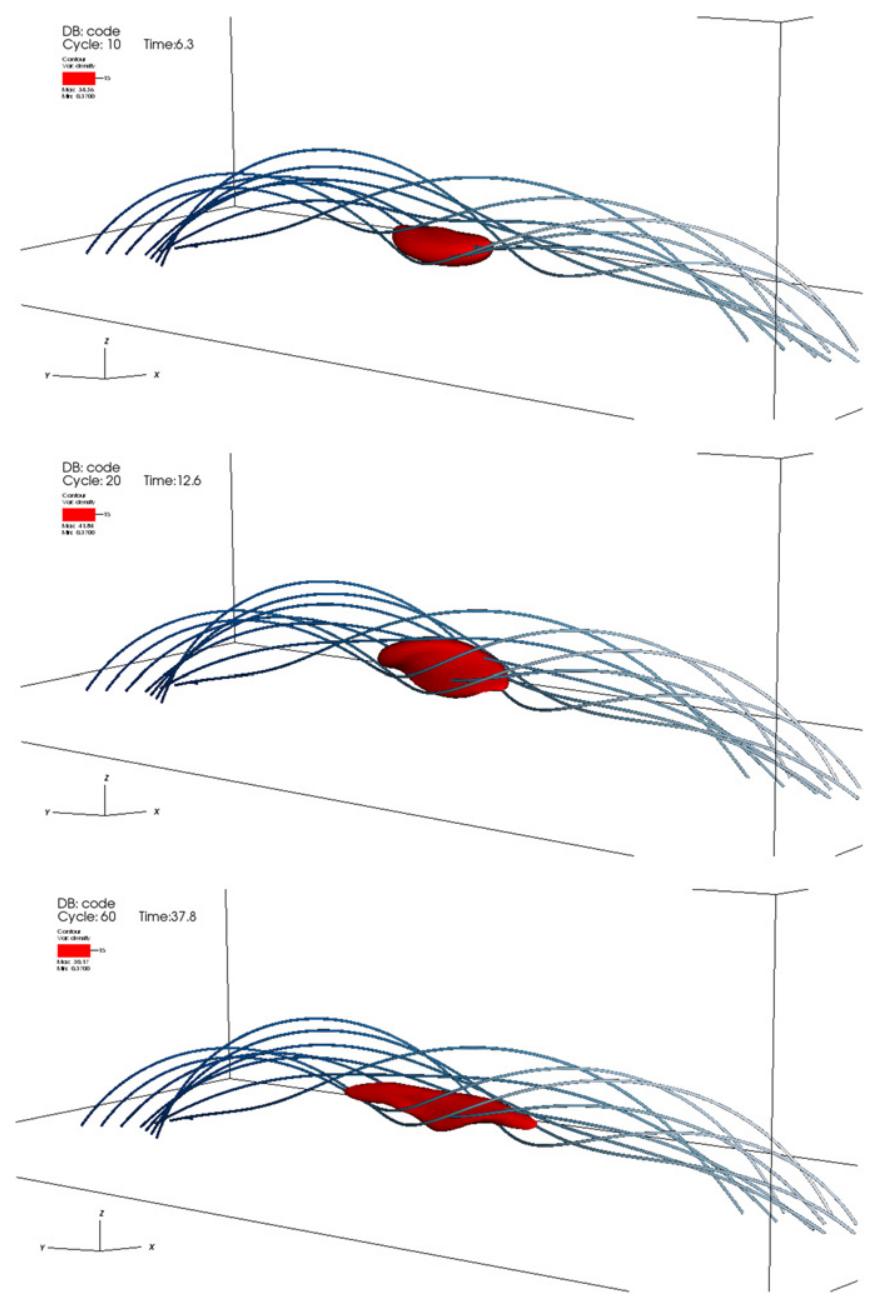

Figure 3. Snapshots of the flux rope configuration plus the prominence at three different times. For this case $N_{t}=8.5$, and the spatial resolution is $600 \mathrm{~km}$.

(An animation of this figure is available.)

obtain a suspended prominence although there are no magnetic dips. The mass does not fall along the curved magnetic field lines because an overpressure is established between the prominence and the base of the corona. This overpressure inevitably produces an increase in temperature in the coronal medium, which is at most $10 \%$ of the coronal temperature (1 MK), but radiative losses and conduction, not present in our model, would try to reduce this temperature increase. This particular support of the prominence is the direct consequence of the use of line-tying conditions at the bottom boundary because pressure perturbations are not allowed to leave the system. In the low- $\beta$ regime gas pressure changes are strongly localized along the magnetic field lines, and the prominence behaves like a piston that moves downward until it reaches an equilibrium state.

For the spatial resolution used in the previous simulations $(600 \mathrm{~km})$, the configurations for moderate and weak twist shown in Figure 4 are able to get to a situation that is close to a quasi-stationary state. For these two cases the newly achieved configuration could be used as a background equilibrium in studies of, for example, transverse or longitudinal oscillations. 

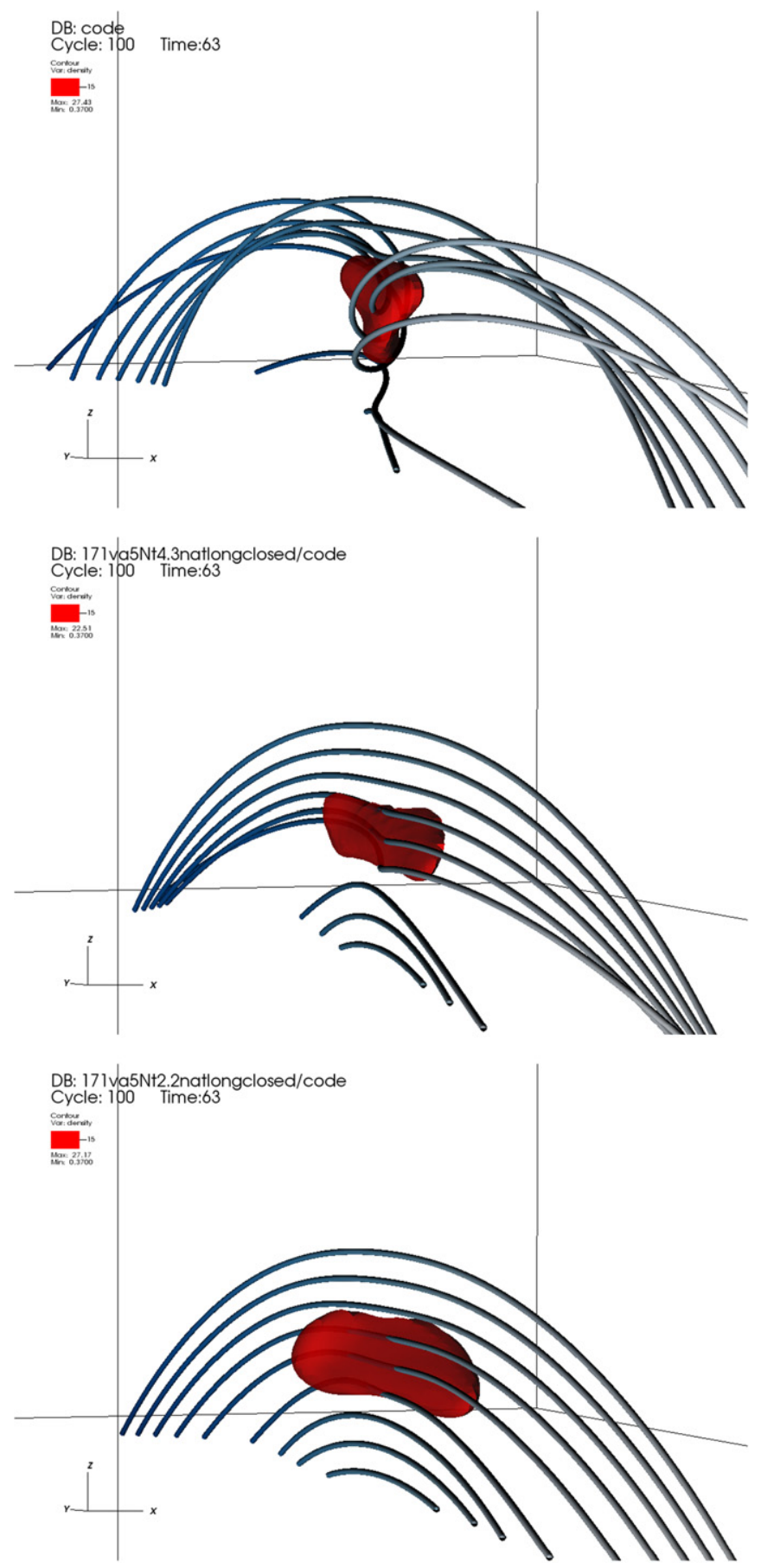

Figure 4. Snapshots of the flux rope configuration plus the prominence at the same instant for the cases $N_{t}=8.5$ (top panel), 4.3 (middle panel), and 2.2 (bottom panel). The KHI is absent here because the spatial resolution is not high enough $(600 \mathrm{~km})$.

\subsection{Time Evolution of Center of Mass}

A detailed analysis of the changes in the prominence reveals that it is in fact quite dynamic, involving rather complicated motions before the quasi-stationary situation is reached. In order to quantify the $3 \mathrm{D}$ movement of the prominence, its center of mass (CM) has been calculated. The computation of this magnitude has been done using the $3 \mathrm{D}$ information of the density in a box that includes the prominence body only, avoiding a significant contribution from the background

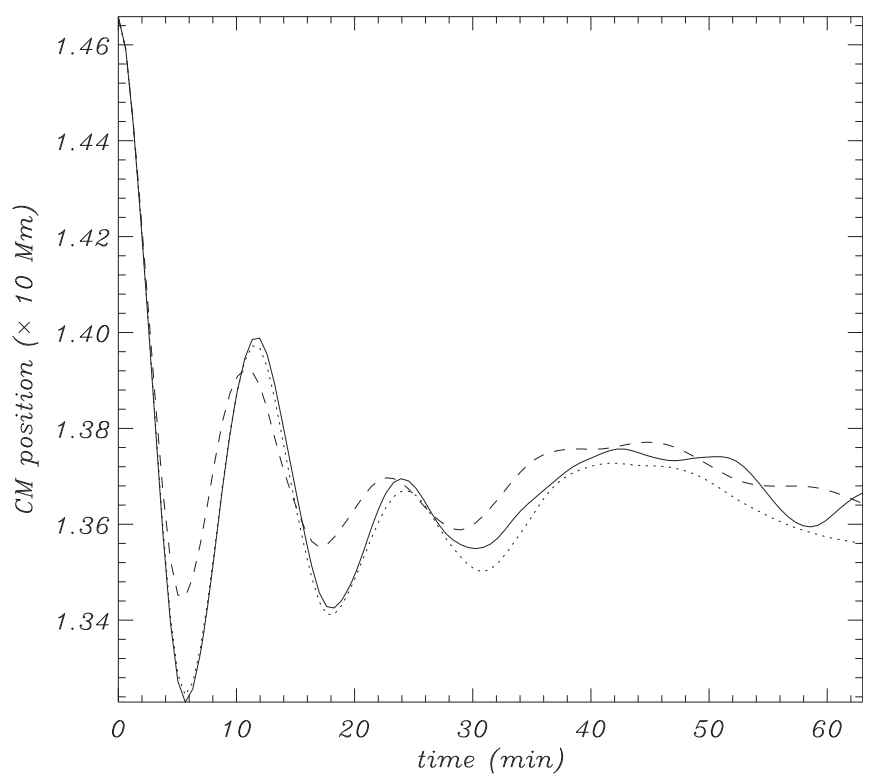

Figure 5. Position of the CM for three different grid resolutions, $1200 \mathrm{~km}$ (dashed line), $600 \mathrm{~km}$ (dotted line), and $300 \mathrm{~km}$ (solid line). For this case $N_{t}=8.5$.

stratified atmosphere present in the whole computational domain. The calculations have been performed using the visualization tool VisIt (Childs et al. 2012, p. 357)

Owing to the symmetry of the system, the $x$ - and $y$ coordinates of the CM are equal to zero, while the $z$-coordinate changes with time. The evolution of this component is represented in Figure 5 and provides a proxy for the global vertical motion of the prominence. In this plot (see solid line), we can distinguish several oscillations. Initially, the prominence descends, but around $t=6$ minutes it starts to displace in the opposite direction, indicating an upward motion. Later, the direction of the motion is reversed several times. This oscillatory pattern lasts for about two periods, i.e., 35 minutes. The characteristic period is, for this simulation, around 12 minutes. The periodicity of the CM reflects a global vertical oscillation of the prominence.

Figure 6 shows the position of the $\mathrm{CM}$ as a function of time for the different values of twist but for a longer run (about $2 \mathrm{hr}$ ). The structure tends to settle down at essentially the same height, around $13.5 \mathrm{Mm}$ for weak and moderate twists. For the case with strong twist the prominence still experiences significant changes after $1 \mathrm{hr}$ of evolution. Surprisingly, the periodicities during the initial relaxation process, from $t=0$ to $t=40$ minutes, are very similar for the three configurations, meaning that twist does not significantly affect the period of oscillation of the global standing transverse mode. This is in agreement with the eigenmode results of standing transverse kink oscillations in straight cylindrical tubes with weak magnetic twist (see Ruderman 2007; Terradas \& Goossens 2012; Ruderman \& Terradas 2015). Furthermore, the behavior found for $N_{t}=2.2$ and 4.3 after 60 minutes of evolution reveals that these configurations are close to a quasi-stationary state.

Interestingly, for the three values of magnetic twist the attenuation of the $\mathrm{CM}$ height with time (in the range $t=0-40$ minutes) is quite strong. The attenuation or damping is a topic that has been discussed extensively, for example, in the context of coronal loop oscillations. In particular, the attenuation in ideal MHD can be associated with the process of 


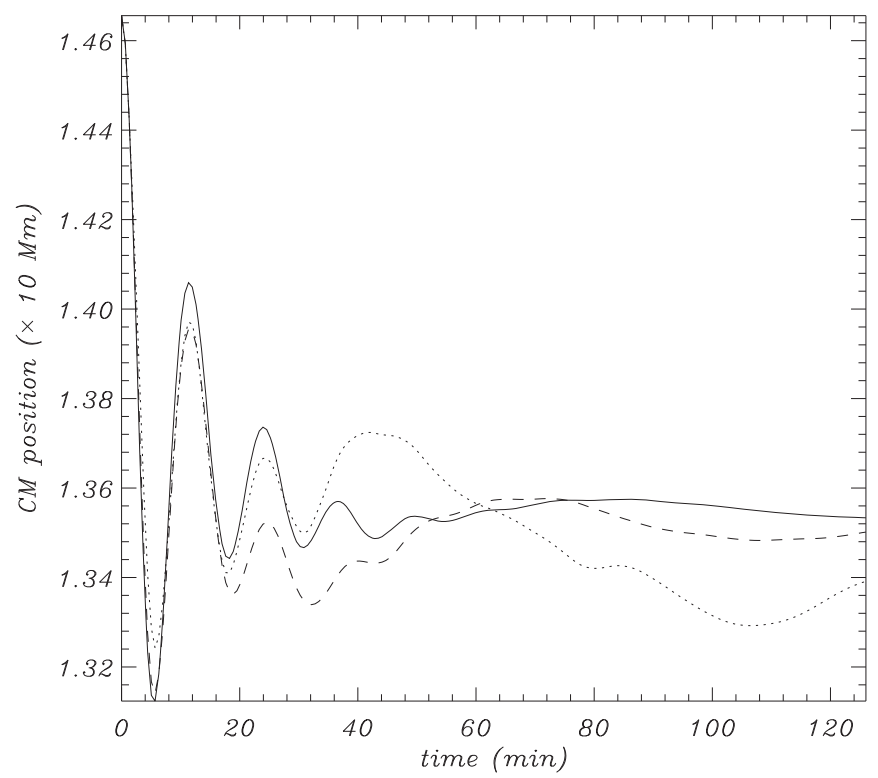

Figure 6. Position of the CM for three different values of the twist, $N_{t}=8.5$ (dotted line), 4.3 (dashed line), and 2.2 (solid line). In these simulations the resolution is $600 \mathrm{~km}$.

resonant absorption and/or wave leakage. Nevertheless, dissipation due to the numerical scheme used in the codes can also be a source of dissipation that is very often disregarded. In order to quantify the potential effect in our simulations and in particular on the attenuation of the position of the CM, we have performed a convergence test and have calculated the CM height for three different grid resolutions. According to Figure 5, convergence of the results is achieved for resolutions between $600 \mathrm{~km}$ (dotted line) and $300 \mathrm{~km}$ (solid line), since the two corresponding curves are not significantly different. The attenuation is not highly affected by the resolution as long as we use a grid separation smaller than $600 \mathrm{~km}$. A resolution of $1200 \mathrm{~km}$ leads to shorter periods and faster attenuation of the global displacement and is not suitable to perform quantitative studies. We can, therefore, claim that the attenuation of the CM position is real (physical) and not dominated by numerical dissipation. This does not mean that at a certain time in the evolution numerical dissipation can be relevant. This issue is discussed in the following sections.

\section{ENERGY CONSIDERATIONS AND DAMPING}

\subsection{Closed Boundaries}

To clarify the origin of the attenuation of the CM position, the energy of the system is investigated. Closed boundary conditions (see Section 3) are first considered (the simulations described so far made use of these conditions). This means that energy is unable to escape from the computational box, and ideally the total energy of the full system should be conserved since the governing equations do not include explicitly dissipative mechanisms. However, numerical dissipation can produce an energy loss. In Figure 7 the different contributions to the total energy are represented as a function of time for the most simple configuration $\left(N_{t}=2.2\right)$. These energies are integrated over the whole 3D domain and have been normalized to the total energy at $t=0$. From the plot we find that since the plasma- $\beta$ is low, the magnetic energy $\left(E_{B}\right)$ has the largest contribution, more than $90 \%$ of the total energy. The

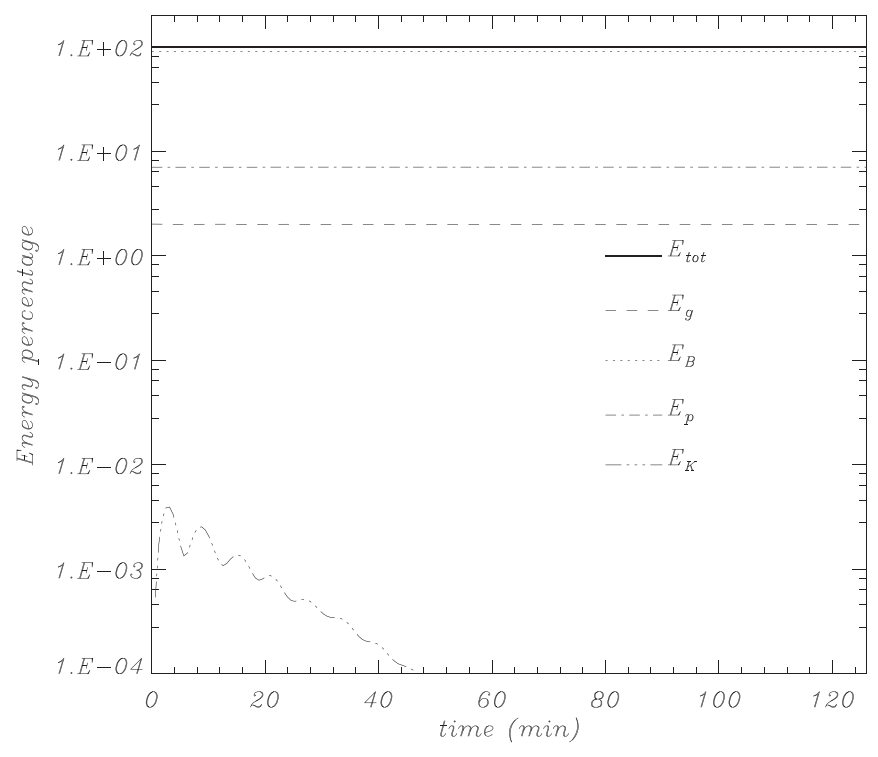

Figure 7. Total energies as a function of time. Note the logarithmic scale in the vertical axis. In this simulation the resolution is $600 \mathrm{~km}$ and $N_{t}=2.2$.

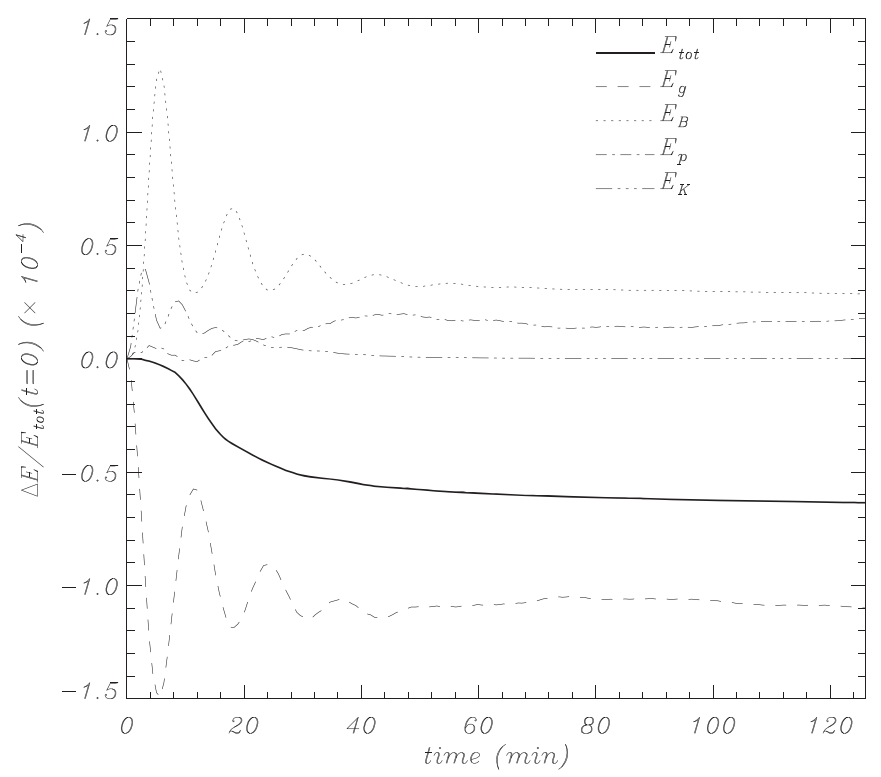

Figure 8. Percentage of differences of energies with respect to their values at $t=0$ as a function of time. Based on the results of Figure 7.

internal energy $\left(E_{p}\right)$ represents about $8 \%$, while the gravitational $\left(E_{g}\right) 2 \%$. The kinetic energy $\left(E_{K}\right)$ is rather small in comparison with the total energy budget $\left(E_{\mathrm{tot}}=E_{B}+E_{p}+\right.$ $E_{g}+E_{K}$ ) of the system. Nevertheless, this kinetic energy accounts for all the motions in the prominence.

From Figure 7 it seems that the total energy is constant with time because of the scale used in the vertical axis of the plot. A more elaborated analysis reveals that indeed part of the total energy is lost. In Figure 8 we have now represented the energy differences with respect to the initial state normalized to the total energy at $t=0$, allowing a better interpretation of the results. The magnetic and internal energies for large times increase with respect to the initial value, while the gravitational energy decreases. This agrees with the global motions described before; the prominence position decreases with time, while it oscillates and settles at a lower height, compressing the 


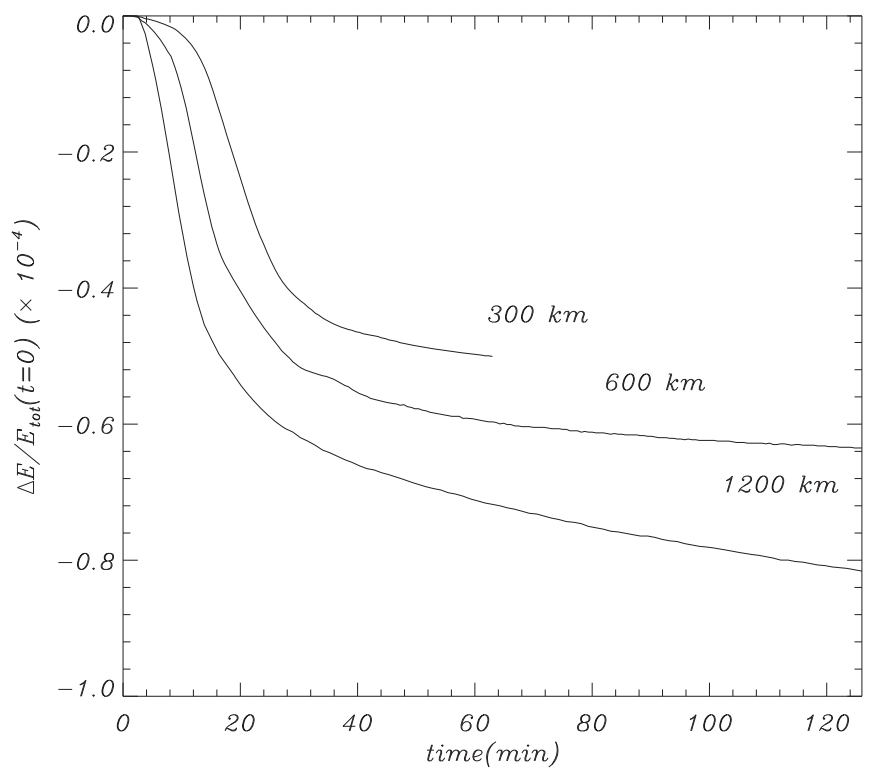

Figure 9. Change in total energy for different grid resolutions. In this simulation $N_{t}=2.2$.

magnetic field and increasing the gas pressure. The kinetic energy initially increases (when the prominence starts to move downward), but soon it decreases until it reaches very low values. More importantly, from the curve of the total energy differences we realize that after around $t=10$ minutes there is a sudden decrease followed by a quite stationary situation. This is a consequence of the development of small scales below the spatial grid resolution and the effect of numerical dissipation. This is corroborated by performing the same plot for different grid resolutions (see Figure 9). The better the resolution, the later the decrease in energy, since smaller scales are resolved. The curves in Figure 9 do not converge to the same value. This is because when the spatial resolution is increased, the overall numerical dissipation is smaller, meaning that globally less energy is dissipated. Ideally, one would expect the change in energy to go to zero as the grid resolution is increased.

It is necessary to remark that this energy loss does not significantly affect the damping time since we have already demonstrated that increasing the resolution gives essentially the same attenuation (see Figure 5). This means that the attenuation in our system is not related to the numerical energy loss and has a physical origin. Note also how the oscillations are very clear in Figure 8 and for the kinetic energy have a period that is half of that of the vertical oscillation since $E_{K}$ varies quadratically with the velocity.

With the aim of understanding the physical origin of the attenuation of the CM position with time, we have performed a simulation for a prominence with a very low density contrast. In this specific simulation we chose a value of 5 for the density enhancement, which is certainly unrealistic, but it will shed light on the damping mechanism. The reason for decreasing the density contrast is that this allows us to study a prominence with a very small mass. Such a light prominence will not experience large motions in the vertical direction in comparison with the previous simulations, and hence nonlinear effects, discussed in the following section, are expected to be very weak. The results of the simulation are shown in Figure 10. The basic period of oscillation, around 8 minutes, is shorter than in Figure 6 (because the prominence is lighter), and the

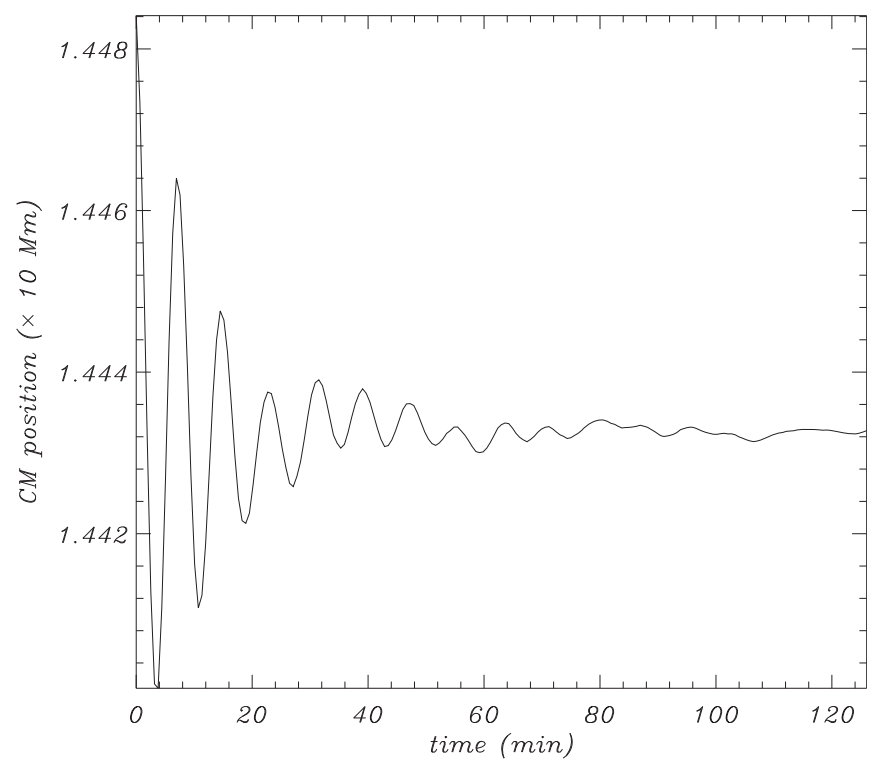

Figure 10. Position of the CM for the case with a low prominence-corona density contrast. For this case $N_{t}=2.2$, and the resolution is $600 \mathrm{~km}$.

attenuation is weaker (now we can count more than nine periods). There is also evidence in Figure 10 of a longer period, which is around 43 minutes. The physical meaning of this periodicity is related to a second motion of the structure of different origin from the vertical oscillation. It corresponds to a global motion but along the magnetic field, and owing to the symmetry in the system, this oscillation is antisymmetric with respect to the center of the prominence, meaning that it produces contractions and dilatations of the whole prominence. In terms of MHD waves it corresponds to a slow antisymmetric mode, and this explains the long periodicity since slow modes are essentially driven by pressure forces, which in our case are rather weak (low- $\beta$ regime). This mode of oscillation is also responsible for the long periodicities found in Figure 6 for times longer than 40 minutes, referred to before as the quasistationary situation. In fact, this periodicity is present from $t=0$ and is superimposed on the shorter periodicity associated with the global vertical oscillation.

We return to the issue of the damping of the vertical oscillation. A comprehensive analysis of the results for the light prominence indicates that the reason for the attenuation is due to the conversion of energy of the transverse vertical motion into localized motions at the lateral edge of the prominence. This is equivalent to the continuum damping mechanism that has been investigated in simpler configurations (such as plasma slabs or magnetic cylinders). In our simulations, although the geometry is complicated, one can identify the locations where there is an increase in kinetic energy due to this process. In Figure 11 density isocontours, representative of the shape of the prominence, are plotted together with a specific isocontour of kinetic energy. It is in a thin region near the edges of the prominence, essentially at the PCTR, where the energy is transferred and concentrated. The power spectrum of the vertical velocity component calculated at different locations provides additional information relevant to the resonant absorption process. Two points localized at the central plane $(x=0)$, one close to the center of the prominence (point 1) and the other at the PCTR (point 2), have been selected. The corresponding power spectra are plotted in Figure 12. At point 

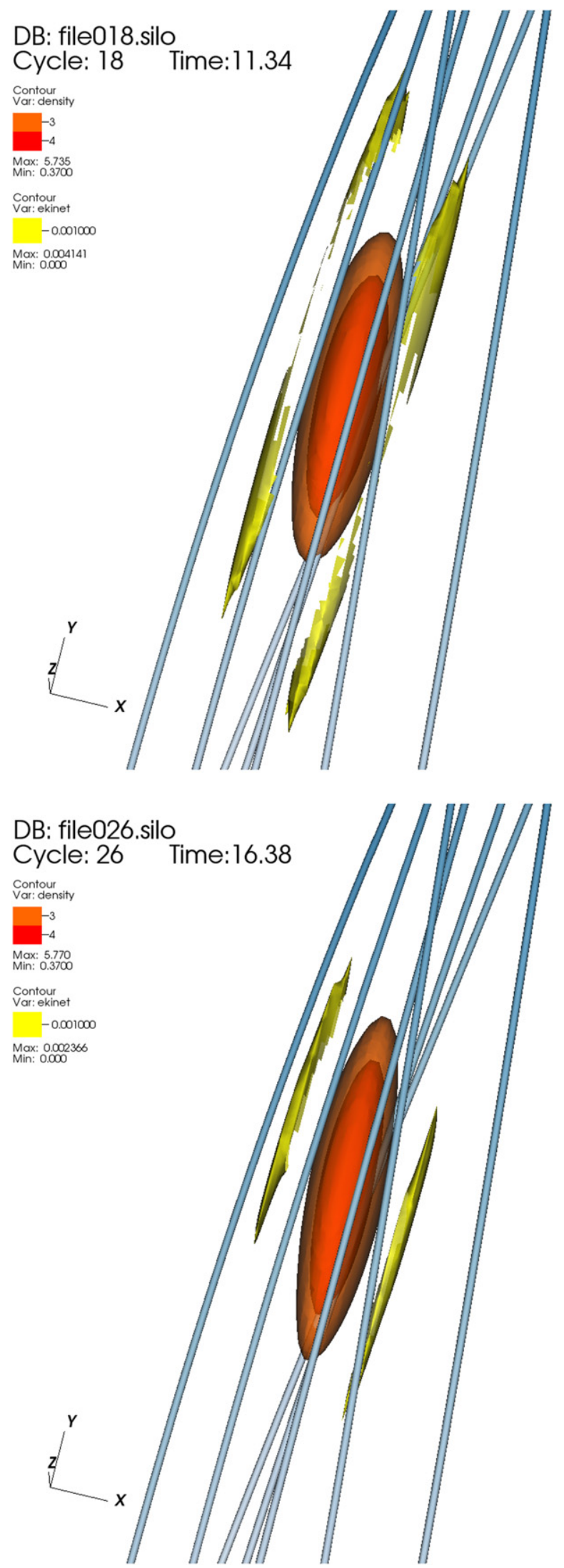

Figure 11. Detail of the prominence (red-orange colors) with some magnetic field lines and the locations where the kinetic energy increases (yellow colors) as a result of continuum damping. For this case $N_{t}=2.2$.

(An animation of this figure is available.)

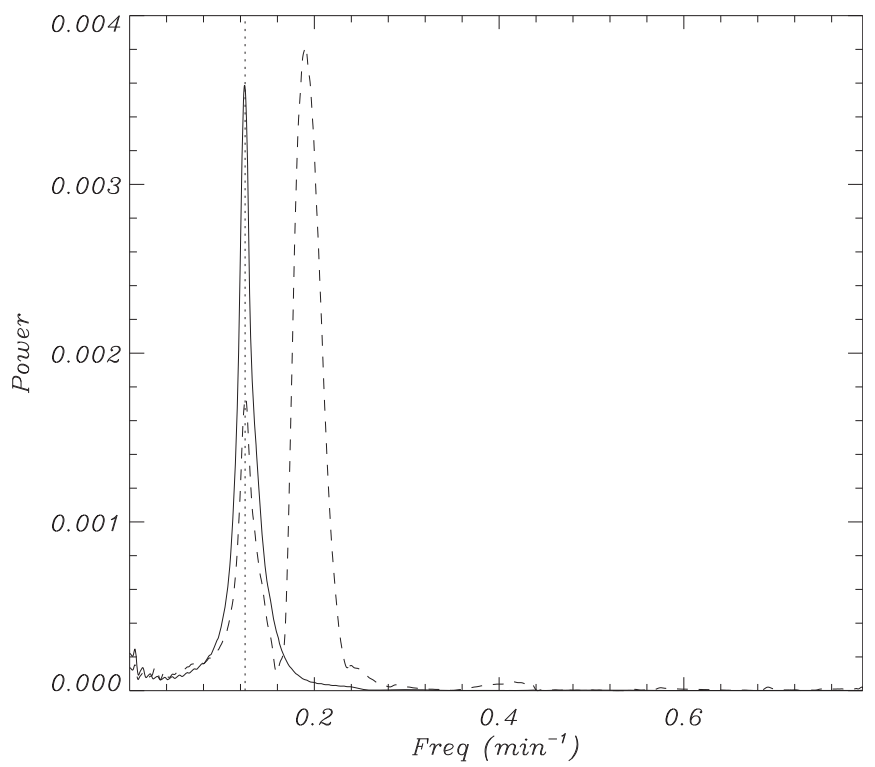

Figure 12. Power spectrum of the vertical velocity component at two different positions. The solid line corresponds to the point close to the center of the prominence, while the dashed line is in the PCTR. The two power spectra show a common peak (plotted with a vertical dotted line), associated with the global motion of the prominence (quasi-mode).

1 the signal has a dominant periodicity of 8 minutes. For point 2 , two peaks are clearly discernible, one located around 8 minutes and the other around 5.25 minutes. Since the two points share a common period, we associate this periodicity with the global mode. This period agrees with the one found in Figure 10 and corresponds to the global vertical oscillation of the prominence. For point 2 , the secondary peak corresponds to a particular Alfvén mode that belongs to the Alfvén continuum. If we chose another point in the PCTR, the frequency spectrum shows power at a different Alfvén frequency. Terradas et al. (2008b) already found this behavior in a rather complicated multistranded loop geometry. In their Figure 3 the signal at a given point shows the collective or global frequency and also the local Alfvén frequency. The amplitude of the global mode decreases with time, while the amplitude of the local Alfvern modes increases with time, owing to the energy transference.

The excitation of local Alfvén modes is also clear in driven problems. For example, Wright (1992), using a simple model, demonstrated how, under different driving conditions, resonant and nonresonant Alfvén modes can be excited in the system (see also De Groof et al. 2002; De Groof \& Goossens 2002). In fact, some of the results of our simulations can be understood assuming that the global mode acts as a driver at a given frequency of finite duration. Wright (1992) showed that for a magnetic field line where the continuum frequency is different from the driving frequency the field line responds at two frequencies: the natural Alfvén frequency and the driven frequency. This is exactly what Figure 12 shows for point 2. In contrast, for a magnetic field line close to the center of the prominence, like for point 1 , the frequency of the driver is essentially the same as the natural Alfvén frequency, and that is why the power spectrum shows a single peak.

To summarize, in the time-dependent problem studied in our simulations, the initial global oscillation of the whole prominence is transferred to the Alfvén continuum modes of the PCTR, providing compelling evidence of the presence of 
continuum damping. This energy transference is most efficient where the frequency of the global mode matches the local Alfvén frequency, but there is also energy transference to neighboring field lines that have their own Alfvén frequency. The amplitude of the Alfvén continuum modes grows with time until all the energy of the global mode has been transferred. Subsequently, the continuum modes oscillate with their natural frequencies. Since the frequency of the Alfvén modes changes with position, this enhances the phase-mixing process. Small spatial scales are generated and eventually smoothed by the numerical dissipation of the scheme.

\subsection{Open Boundaries}

Now open boundary conditions are considered, allowing the role of energy leakage and its contribution to the observed attenuation of the position of the CM to be investigated. The results indicate that for the present configuration the energy loss due to wave leakage through the boundaries is rather small since the position of the CM is essentially the same as in the situation for closed boundaries. Thus, wave leakage is not the main cause of damping of the vertical oscillations. The dominant physical process is transfer of energy to Alfvén oscillations of neighboring field lines.

There is additional indirect evidence about the absence of significant leakage in our system. From the calculation of the power spectrum at different points, described before, and the inferred period of the global oscillation, wave leakage of this mode is not possible from the theoretical point of view. The cause is that the Alfvénic modes in the coronal environment outside the prominence have frequencies that are above the frequency of the global mode. This is incompatible with the properties of leaky modes, which are fast magnetoacoustic modes with frequencies above the local Alfvén frequency and therefore have a propagating nature. This propagating feature is precisely the one responsible for the attenuation of the mode since the energy of the global mode is emitted away. These types of modes are not possible in our configuration.

\section{KELVIN-HELMHOLTZ INSTABILITY}

The results of the previous section indicate that resonant absorption is responsible for the attenuation of the position of the CM. This is true at least for the case of the light prominence. The results for the typical prominence considered in our simulations (see Figure 6) also suggest a strong damping. However, in this last case the physics involved in the attenuation is more intricate, especially owing to nonlinear phenomena. The reason is that since now the prominence is more massive, the vertical motions and therefore the shear motions at the PCTR have larger amplitudes and ultimately produce a KHI type. This instability operates in the absence of inhomogeneous layers, i.e., for a discontinuous change in the velocity, but also under the process of resonant absorption and the associated phase mixing. A clear indication of the phasemixing process before the onset of the instability is shown in the top panel of Figure 13, where the $y$-component of the vorticity is plotted in the plane $y=0$. The essentially vertical bands are due to the generation of small length scales, and their number increases with time as the process of phase mixing develops. In fact, these vorticity layers seem to propagate across the prominence structure, but this is just an apparent propagation due to the existence of a continuum of Alfvén
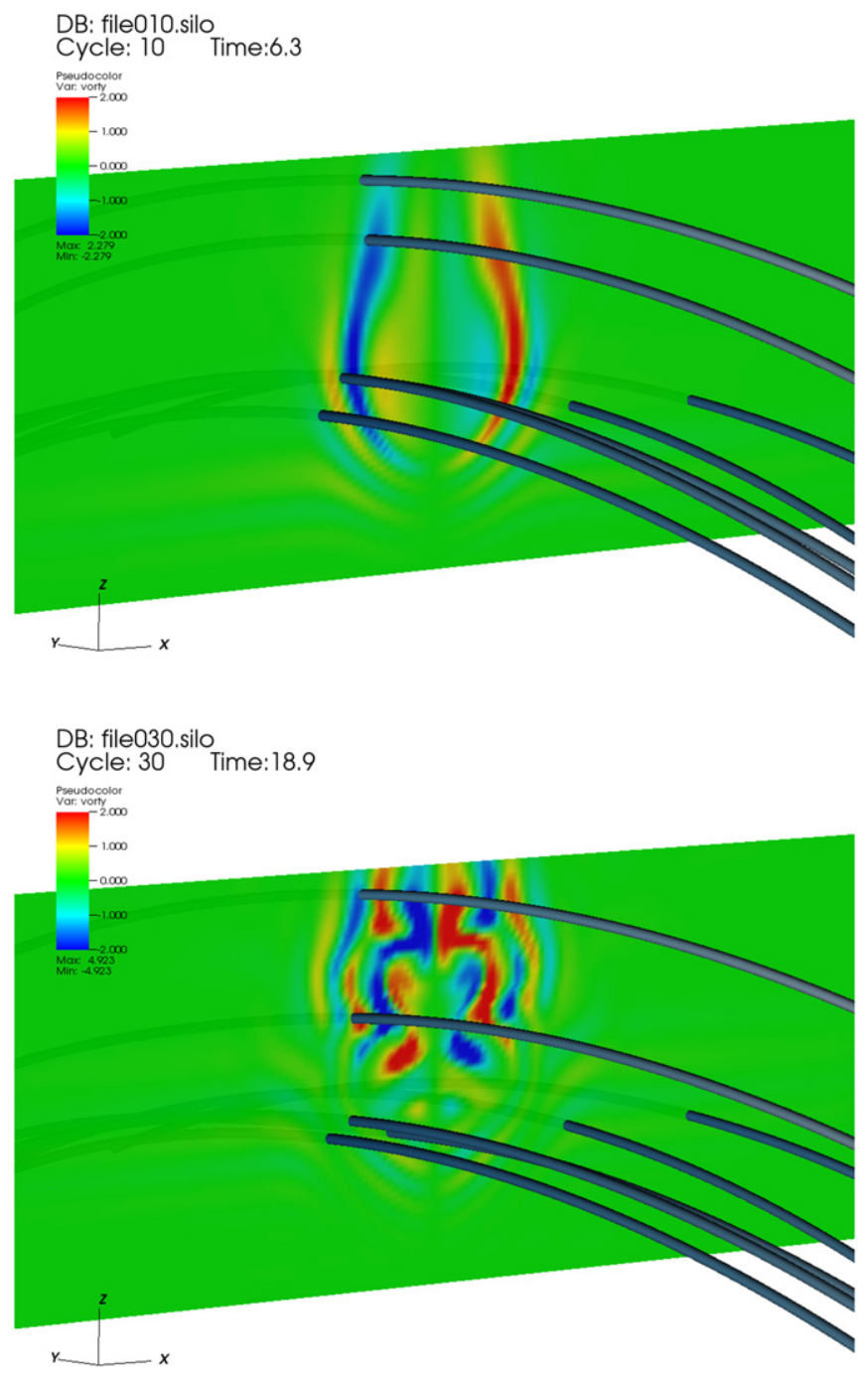

Figure 13. Detail of the $y$-component of the vorticity $(\nabla \times v)$ in the plane $y=0$. For this case $N_{t}=2.2$ and the spatial resolution is $300 \mathrm{~km}$.

waves (see, e.g., Kaneko et al. 2015). At some instant they become unstable to the KHI, and this happens on a timescale that is less than the period of the global vertical oscillation of the structure. This stage of the nonlinear evolution is identified in the bottom panel of Figure 13. Note that the most likely place for the KHI to occur is where the velocity shear is largest and the magnetic field is perpendicular to the velocity (e.g., Rankin et al. 1993). For the fundamental standing transverse mode this is precisely the antinode of the velocity, located at half the flux rope length, i.e., in the plane plotted in Figure 13.

This nonlinear problem, i.e., the development of the KHI in a phase-mixed layer, has been addressed analytically by Heyvaerts \& Priest (1983) and Browning \& Priest (1984) (see also Allan \& Wright 2010). More recently, Terradas et al. (2008a) and Antolin et al. (2015) have studied the KHI numerically using straight cylinders with nonhomogeneous layers in the radial direction, while Soler et al. (2010b) have performed an analytic study assuming a jump in the velocity shear. The flux rope configuration studied in the present work, although more complex than the simple straight tube, is not an exception. The development of the instability is quite evident in the simulations with the highest resolution, like the one shown in Figure 13. For the intermediate spatial resolution $(600 \mathrm{~km})$ it 

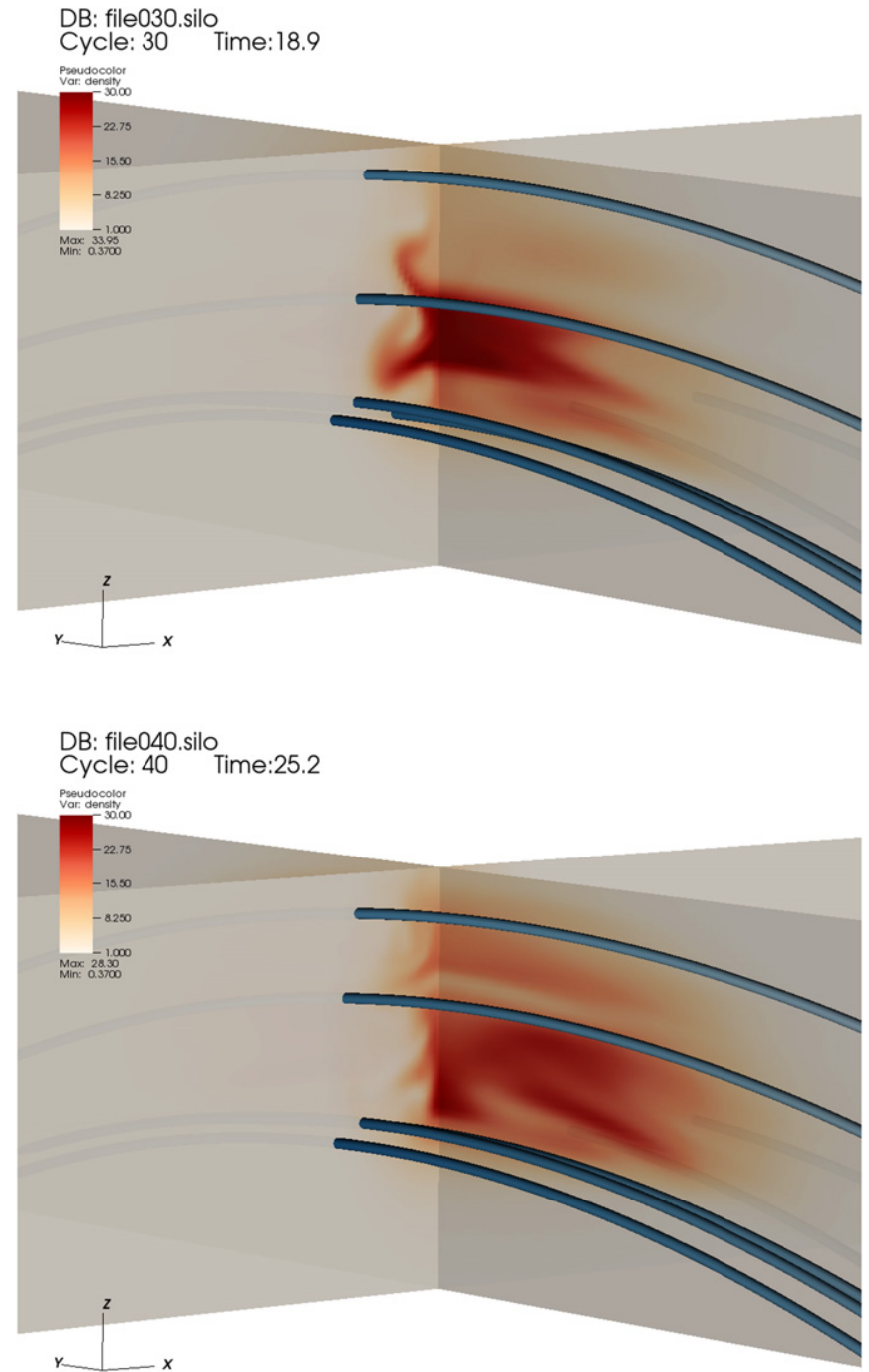

Figure 14. Detail of the density evolution at two perpendicular planes. For this case $N_{t}=2.2$ and the spatial resolution is $300 \mathrm{~km}$.

is not so obvious. Further evidence for the instability is found in Figure 14 for the case $N_{t}=2.2$, where the density distributions at two perpendicular planes that intersect at the center of the prominence are plotted together with some selected magnetic field lines. The initially compact shape of prominence progresses, and ripples at the sides of the prominence start to appear (see top panel and the associated vorticity plot, bottom panel of Figure 13). These are precisely the locations where the velocity shear is strong. Later, small scales are still developing, and some structuring is devised along the field lines (see bottom panel of Figure 14).

It is interesting to use the ideas developed in Browning \& Priest (1984) and Allan \& Wright (2010) in the context of our simulations. Allan \& Wright (2010) suggested a method to estimate the time at which the growth of the KHI in the phasemixing layer becomes significant. Based on the results of Browning \& Priest (1984), Allan \& Wright (2010) defined the normalized maximum growth rate (see their Equations (3) and (6)) as

$$
\Gamma_{\mathrm{KH}} \approx \frac{1.7 V_{0}}{4 \omega_{\mathrm{A}} L_{\mathrm{ph}}},
$$

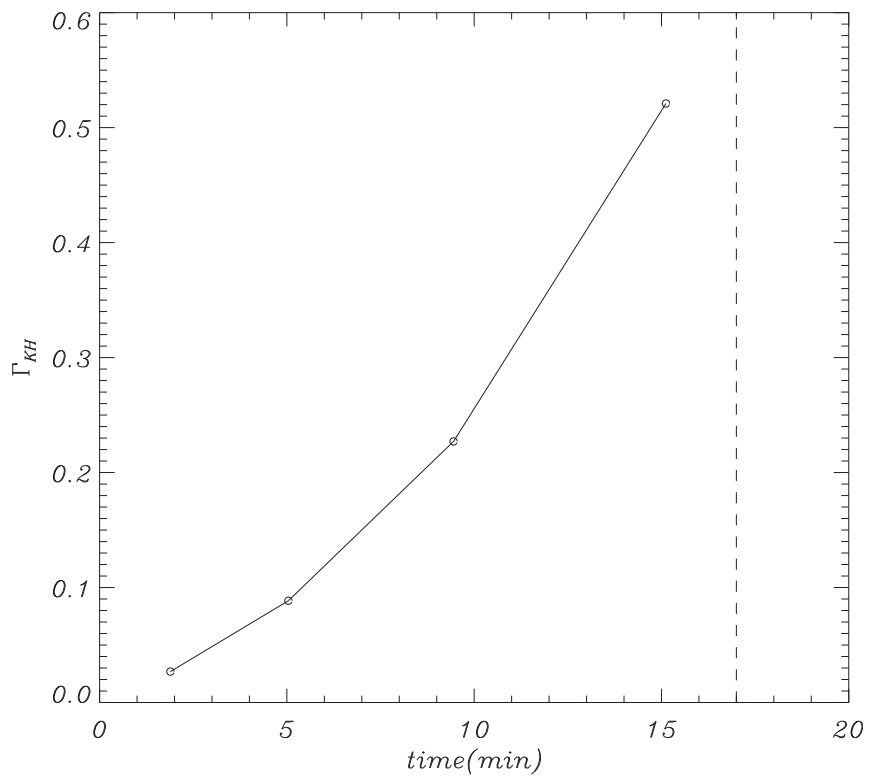

Figure 15. Normalized maximum $\mathrm{KH}$ growth rate, $\Gamma_{\mathrm{KH}}$, as a function of time for the simulation shown in Figure 14. The dashed line corresponds to the time estimation of the onset of the KHI from the simulations.

where $V_{0}$ is the wave amplitude, $\omega_{\mathrm{A}}$ the frequency at the resonance, and $L_{\mathrm{ph}}$ the phase-mixing length. These three magnitudes can be estimated from the simulations. The amplitude depends on time since energy is pumped into the layer, and $L_{\mathrm{ph}}$ is also time dependent since the phase-mixing process continuously generates smaller length scales with time (we have used the distance between two maxima as an estimation for $L_{\mathrm{ph}}$ ). This means that $\Gamma_{\mathrm{KH}}$ is also a function of time. Allan \& Wright (2010) defined the normalized growth rate of the instability in such a way that the growth of the KHI during a quarter cycle of a large velocity shear will be significant if $\Gamma_{\mathrm{KH}}$ is comparable to or greater than unity. The results of the calculation of $\Gamma_{\mathrm{KH}}$ based on our simulations at the early stages of the time evolution are shown in Figure 15. This magnitude is much less than 1 at the very beginning of the simulation, but it shows a rapid increase with time. The trend of the curve indicates that values of the order of 1 are possible after 15 minutes of evolution. This agrees with the timing of the onset of the instability inferred from the simulation, which is around $t=17$ minutes (see dashed vertical line in Figure 15). The match with the analytic prediction is good, and our simulations suggest that $\Gamma_{\mathrm{KH}} \approx 0.5$ is sufficient for the KHI to take over.

Another question that arises here is whether the attenuation, discussed in the previous section for a light prominence, is produced by resonant absorption at the inhomogeneous layers, or if it is instead more related to the nonlinear effects that generate the vortical structures at the sides of the prominence due to the KHI. In other words, the KHI by itself may provide a mechanism to attenuate the prominence even in the absence of resonant absorption since this instability precisely takes energy from the bulk flow (the transverse vertical oscillation in our case) that eventually cascades to smaller scales. Depending on the amplitude of the oscillation (the KHI is a nonlinear problem, while resonant damping works linearly), the KHI could transfer the energy more efficiently than resonant 

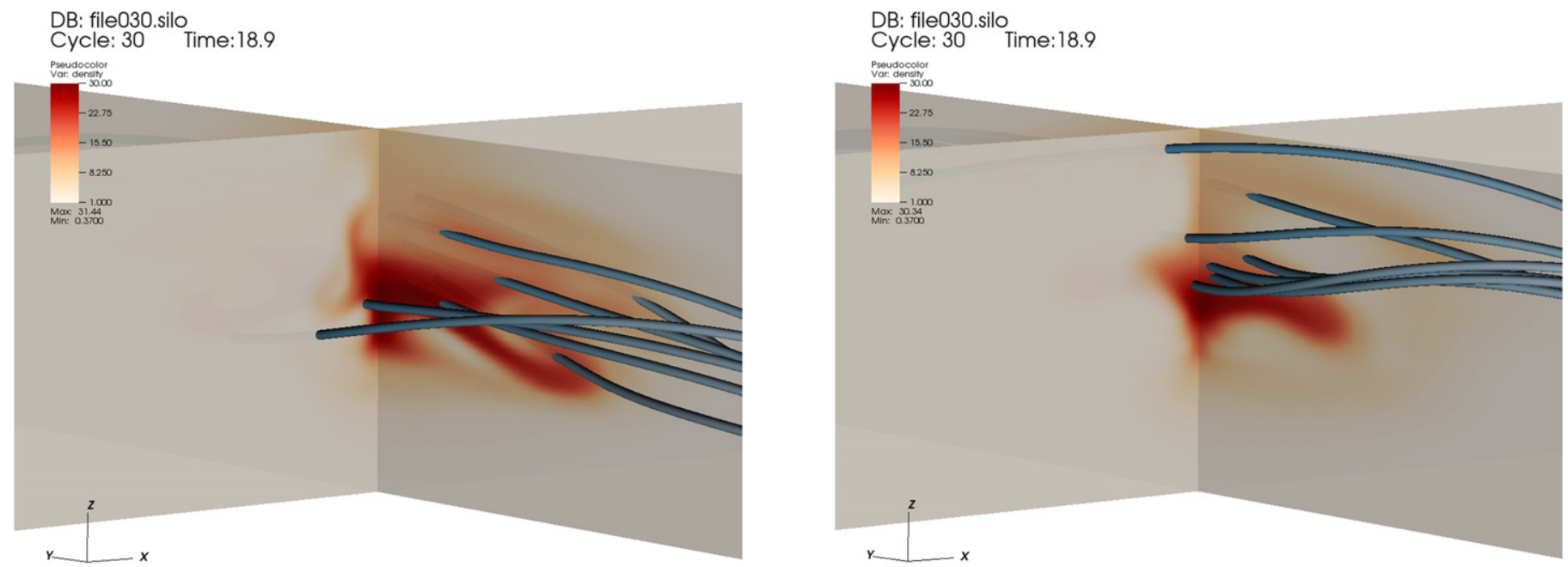

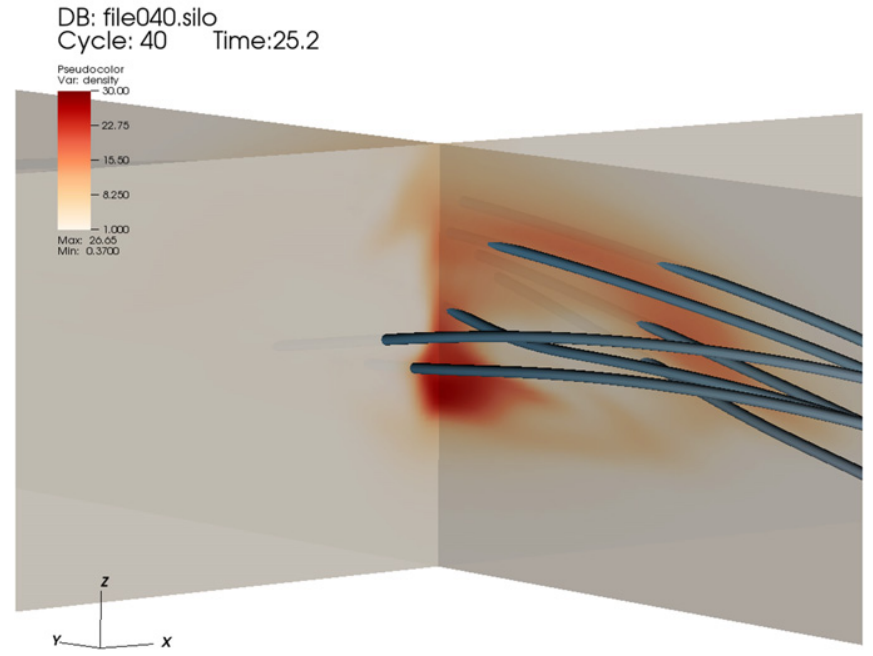

Figure 16. Same as in Figure 14 but for $N_{t}=4.3$.

absorption. This is an attractive problem that requires a careful analysis, preferably using a simpler model like the straight cylindrical tube, and is left for future studies. In this respect, Ruderman et al. (2010) have proposed that the nonlinear coupling between kink and fluting modes may accelerate the damping of the oscillations since fluting modes have a faster attenuation rate.

Finally, it is interesting to investigate the effect of twist on the development of the KHI for the highest spatial resolution. In Figures 16 and 17, the density distributions for the configurations with moderate and strong twist are plotted. We see that the ripples associated with the instability develop in a different way; for moderate twist they are clearly visible at intermediate times (top panel of Figure 16). These vortical structures are not so evident in Figure 17, representing the situation with the strongest twist, but a detailed inspection of the motions shows that the instability is still present. It is known that magnetic twist must have a stabilizing effect (see Soler et al. 2010b), but in the present configuration, even for the situation named here as strong twist, it is still too weak to inhibit completely the development of the instability. A distinctive feature of the flux rope with weak twist, showing quite a compact shape, in comparison with the situations of

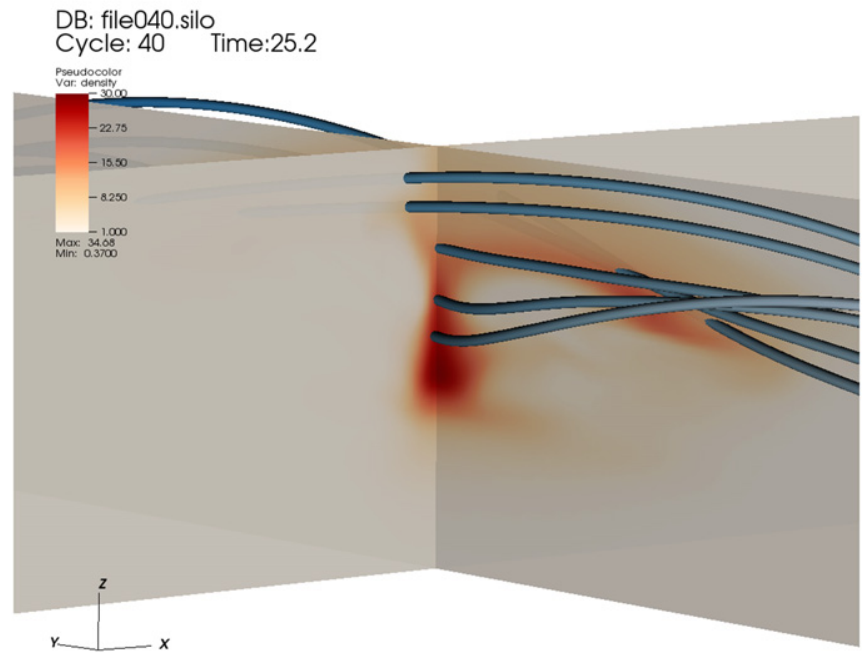

Figure 17. Same as in Figure 14 but for $N_{t}=8.5$.

moderate and strong twist, is that in these last two cases elongated structures along the flux rope axis are generated (see bottom panels of Figures 16 and 17). These structures are lying mainly horizontally with respect to $z=0$. This behavior is due to the fact that twist introduces local dips and mass tends to accumulate around these dips. However, at the center of the flux rope, even in the case with the strongest twist, the magnetic field lines are essentially horizontal, and therefore the dense material does not reorganize much along these lines, producing the horizontal structuring.

\section{CONCLUSIONS AND DISCUSSION}

The time evolution of a cold prominence initially embedded in a magnetic flux rope has revealed interesting characteristics of the structure. We have concentrated on sustained prominences, i.e., detached from the photosphere. The different numerical simulations have shown how the magnetic field evolves in response to the dense prominence that is pulled downward by gravity. The various twisted flux ropes considered in this work, together with the embedded prominences, show a different evolution. We have shown that in some cases they are clearly evolving toward a stationary state. These quasistationary $3 \mathrm{D}$ solutions can be used in the future for other 
purposes such as the analysis of winking filaments produced by external perturbations like Moreton waves, EIT waves, or nearby flares and CMEs.

During the relaxation to the quasi-stationary equilibrium, the system goes through several periodic vertical oscillations. In the linear regime, i.e., when the amplitude of oscillation is small in comparison with the local Alfvén speed, the process of continuum damping transfers the excess of energy in the system to the PCTR. It is in this highly inhomogeneous layer where the process of phase mixing takes place. This energy redistribution explains the attenuation of the position of the CM of the prominence body. Thus, at least in the linear regime the resonant absorption process is crucial in the relaxation of the structure. The energy in the PCTR is eventually dissipated by the numerical scheme used to perform the simulation, and this dissipation can be hardly avoided owing to the continuous generation of small spatial scales with time by the phasemixing mechanism. Fortunately, although the numerical dissipation produces an energy loss, this does not necessarily mean that the attenuation of the CM is dominated by this artificial dissipation. In fact, it is known that a small dissipation does not change the damping time (Poedts \& Kerner 1991) of the quasi-mode (i.e., the resonantly damped global mode); it affects the dissipation in the layer but not the global attenuation (see Terradas et al. 2006a, for the linear time evolution with dissipation). The different convergence tests performed in the present study point in this direction. Wave leakage is not relevant in our model and does not contribute to the attenuation of the position of the CM.

The problem studied here demonstrates the necessity of a proper calculation of the Alfvén and the slow continuum in general 3D geometries like the ones considered in the present work. The efforts done so far in the calculation of the eigenproblem for magnetic field resonances in the context of magnetospheric plasmas (see Singer et al. 1981; Rankin et al. 2006; Kabin et al. 2007; Degeling et al. 2010) may shed some light about the application of the known techniques for closed geomagnetic field lines to the problem of coronal loops and solar prominences.

The vertical oscillations found in the simulations are closely related to the eigenmodes (quasi-modes) of the configuration, and therefore the same periodicities should be found by perturbing (in the vertical direction) the stationary equilibrium. Owing to the symmetry in the system, only the vertically polarized mode has been excited in our numerical experiments, but according to previous works, in similar toroidal configurations without magnetic twist (Terradas et al. 2006b; van Doorsselaere et al. 2009) there should be also a horizontally polarized mode with essentially the same period as the vertical mode (this is true in the thin-tube approximation only). Interestingly, the period of the standing vertical oscillation does not change much with magnetic twist, at least for the three configurations studied here. This can have implications from the seismological point of view. The slow antisymmetric mode has been also detected in the simulations, but future studies are required to understand the existence of other modes that, owing to the symmetry of the system, have not been excited in our numerical experiments.

Nonlinearity complicates the physics and thus the interpretation of the outcome of the simulations. We have demonstrated that for the highest spatial resolution $(300 \mathrm{~km})$ the three twisted flux ropes analyzed in this work develop KHI at the PCTR. For all the cases, the attenuation of the CM of the prominence is rather strong, and the shear instability may contribute to this attenuation (in the linear regime it is only due to resonant absorption). A meticulous investigation of the effect of the KHI on the damping is required.

For the values of twist considered here, constrained by observations (the maximum number of turns of the magnetic field is at most two or three for stable configurations), the poloidal component of the magnetic field, which provides an additional stabilizing magnetic force, is unable to completely suppress the shear instability at the PCTR. The evolution of the hosted prominence inside the flux rope suggests the formation of elongated structures lying essentially horizontal and generated by the KHI. These results are in contrast to the situation found in the dipped magnetic arcade studied by Terradas et al. (2015), with the long axis of the prominence situated essentially perpendicular to the magnetic field. In that configuration it was found that the MRT instability is very efficient (see also Hillier et al. 2012a, 2012b). Fingers and plumes evolve at the bottom of the interface between the prominence and corona, producing essentially vertical structures. Ideally, the different morphological observed properties of prominences regarding the presence of either horizontal or vertical structuring could be used to provide hints about the geometry of the corresponding underlying magnetic structure. Horizontal structures are more consistent with magnetic flux ropes, while vertical structuring along the longitudinal axis of the prominence is associated with arcade configurations according to our study.

There are two basic reasons for the absence of the MRT instability in our flux rope configuration. The first reason, and the most important, is the orientation of the prominence with respect to the magnetic field. In the arcade model the prominence is permeated by a perpendicular magnetic field (when magnetic shear is small), while in our flux rope model there is always an important magnetic component along the prominence body. This means that for perturbations along the longitudinal axis, the flux rope prominence is intrinsically much more stable with respect to the MRT instability since magnetic forces produce a stabilizing effect that is missing in the unsheared arcade configuration. Second, for perturbations perpendicular to the longitudinal axis of the prominence, the localized twist provides a magnetic component that tends again to stabilize the structure. This is similar to the effect of shear in the arcade configuration, but with the difference that the stabilizing component changes much more rapidly with position in the flux rope model. This strong variation results in a much more stable configuration (see, e.g., Ruderman et al. 2014).

Finally, it seems clear that line-tying conditions at the base of the corona are too restrictive. These conditions are crucial to obtain suspended prominences and make the effect of gas pressure along the magnetic field lines play a very important role in the support, especially for the cases of low magnetic twist without magnetic dips. However, from observations it is quite obvious that there is a continuous interchange of material with the chromosphere, which is completely missing in our simulations owing to the line-tying conditions. In addition, observations also show that blobs of material falling in the direction of the chromosphere do not seem to stop at a given height as one would expect with these boundary conditions in ideal MHD. The incorporation of more realistic conditions in 
our models including the chromosphere and the pertinent nonideal effects, such as radiative losses and conduction, is of capital relevance.

J.T. and R.S. acknowledge support from MINECO and UIB through a Ramón y Cajal grant. The authors acknowledge support by the Spanish MINECO and FEDER funds through project AYA2014-54485-P. M.L. acknowledges the support by the Spanish Ministry of Economy and Competitiveness through projects AYA2011-24808, AYA2010-18029, and AYA201455078-P. This work contributes to the deliverables identified in FP7 European Research Council grant agreement 277829, "Magnetic Connectivity through the Solar Partially Ionized Atmosphere" (PI: E. Khomenko). M.L., J.T., and J.L.B. also acknowledge support from the International Space Science Institute (ISSI) to the Team 314 on "Large-Amplitude Oscillation in prominences" led by M. Luna. The authors also thank Dr. Titov for providing a Fortran routine to calculate the flux rope magnetic configuration of the Titov \& Demoulin model. The Open Source VisIt is supported by the Department of Energy with funding from the Advanced Simulation and Computing Program and the Scientific Discovery through Advanced Computing Program. We thank the anonymous referee for useful comments and suggestions that helped to improve the paper.

\section{REFERENCES}

Allan, w., \& Wright, A. N. 2010, JGR, 102, 19927

Antolin, P., Okamoto, T. J., De Pontieu, B., et al. 2015, ApJ, 809, 72

Arregui, I., \& Ballester, J. L. 2011, SSRv, 158, 169

Arregui, I., Soler, R., Ballester, J. L., \& Wright, A. N. 2011, A\&A, 533, A60 Arregui, I., Terradas, J., Oliver, R., \& Ballester, J. L. 2008, ApJL, 682, L141 Berenger, J.-P. 1994, JCoPh, 114, 185

Blokland, J. W. S., \& Keppens, R. 2011, A\&A, 532, A94

Browning, P. K., \& Priest, E. R. 1984, A\&A, 131, 283

Cally, P. S. 1991, JPIPh, 45, 453

Chen, L., \& Hasegawa, A. 1974, PhFl, 17, 1399

Childs, H., Brugger, E., Whitlock, B., et al. 2012, in High Performance Visualization-Enabling Extreme-Scale Scientific Insight, ed. E. W. Bethel,

H. Childs, \& C. Hansen (Boca Raton, FL: CRC Press), 357

De Groof, A., \& Goossens, M. 2002, A\&A, 386, 691

De Groof, A., Paes, K., \& Goossens, M. 2002, A\&A, 386, 681

Degeling, A. W., Rankin, R., Kabin, K., Rae, I. J., \& Fenrich, F. R. 2010, JGRA, 115, 10212

Goossens, M., Andries, J., \& Aschwanden, M. J. 2002, A\&A, 394, L39

Goossens, M., Hollweg, J. V., \& Sakurai, T. 1992, SoPh, 138, 233

Grossmann, W., \& Tataronis, J. 1973, ZPhy, 261, 217

Heyvaerts, J., \& Priest, E. R. 1983, A\&A, 117, 220

Hillier, A., Berger, T., Isobe, H., \& Shibata, K. 2012a, ApJ, 746, 120

Hillier, A., Isobe, H., Shibata, K., \& Berger, T. 2012b, ApJ, 756, 110

Hillier, A., \& van Ballegooijen, A. 2013, ApJ, 766, 126

Hollweg, J. V. 1987, ApJ, 312, 880

Hollweg, J. V., \& Yang, G. 1988, JGR, 93, 5423
Hood, A. W., Ruderman, M., Pascoe, D. J., et al. 2013, A\&A, 551, A39 Hu, F. Q. 2001, JCoPh, 173, 455

Ionson, J. A. 1978, ApJ, 226, 650

Kabin, K., Rankin, R., Waters, C. L., et al. 2007, P\&SS, 55, 820

Kaneko, T., Goossens, M., Soler, R., et al. 2015, ApJ, 812, 121

Kuperus, M., \& Raadu, M. A. 1974, A\&A, 31, 189

Lee, M. A., \& Roberts, B. 1986, ApJ, 301, 430

Li, T., \& Zhang, J. 2012, ApJL, 760, L10

Low, B. C., \& Zhang, M. 2004, ApJ, 609, 1098

Luna, M., Knizhnik, K., Muglach, K., et al. 2014, ApJ, 785, 79

Mackay, D. H., Karpen, J. T., Ballester, J. L., Schmieder, B., \& Aulanier, G. 2010, SSRv, 151, 333

Mann, I. R., Wright, A. N., \& Cally, P. S. 1995, JGR, 100, 19441

Okamoto, T. J., Antolin, P., De Pontieu, B., et al. 2015, ApJ, 809, 71

Parchevsky, K. V., \& Kosovichev, A. G. 2007, ApJ, 666, 547

Pascoe, D. J., Wright, A. N., \& De Moortel, I. 2010, ApJ, 711, 990

Poedts, S., \& Kerner, W. 1991, PhRvL, 66, 2871

Poedts, S., Kerner, W., Goedbloed, J. P., et al. 1992, PPCF, 34, 1397

Priest, E. R., Hood, A. W., \& Anzer, U. 1989, ApJ, 344, 1010

Rae, I. C. 1982, PlPh, 24, 133

Rankin, R., Harrold, B. G., Samson, J. C., \& Frycz, P. 1993, JGR, 98, 5839

Rankin, R., Kabin, K., \& Marchand, R. 2006, AdSpR, 38, 1720

Ruderman, M. S. 2007, SoPh, 246, 119

Ruderman, M. S., Goossens, M., \& Andries, J. 2010, PhPl, 17, 082108

Ruderman, M. S., \& Roberts, B. 2002, ApJ, 577, 475

Ruderman, M. S., \& Terradas, J. 2013, A\&A, 555, A27

Ruderman, M. S., \& Terradas, J. 2015, A\&A, 580, A57

Ruderman, M. S., Terradas, J., \& Ballester, J. L. 2014, ApJ, 785, 110

Ryutova, M., Berger, T., Frank, Z., Tarbell, T., \& Title, A. 2010, SoPh, 267,75

Sakurai, T., Goossens, M., \& Hollweg, J. V. 1991, SoPh, 133, 227

Sedláček, Z. 1995, in AIP Conf. Ser. 345 (Melville, NY: AIP), 119

Singer, H. J., Southwood, D. J., Walker, R. J., \& Kivelson, M. G. 1981, JGR, 86,4589

Soler, R., Arregui, I., Oliver, R., \& Ballester, J. L. 2010a, ApJ, 722, 1778

Soler, R., Oliver, R., Ballester, J. L., \& Goossens, M. 2009, ApJL, 695, L166

Soler, R., \& Terradas, J. 2015, ApJ, 803, 43

Soler, R., Terradas, J., Oliver, R., Ballester, J. L., \& Goossens, M. 2010b, ApJ, 712,875

Southwood, D. J. 1974, P\&SS, 22, 483

Tataronis, J., \& Grossmann, W. 1973, ZPhy, 261, 203

Terradas, J., Andries, J., Goossens, M., et al. 2008a, ApJL, 687, L115

Terradas, J., Arregui, I., Oliver, R., et al. 2008b, ApJ, 679, 1611

Terradas, J., \& Goossens, M. 2012, A\&A, 548, A112

Terradas, J., Oliver, R., \& Ballester, J. L. 2006a, ApJ, 642, 533

Terradas, J., Oliver, R., \& Ballester, J. L. 2006b, ApJL, 650, L91

Terradas, J., Soler, R., Luna, M., Oliver, R., \& Ballester, J. L. 2015, ApJ, 799, 94

Titov, V. S., \& Démoulin, P. 1999, A\&A, 351, 707

Török, T., \& Kliem, B. 2003, A\&A, 406, 1043

Török, T., Kliem, B., \& Titov, V. S. 2004, A\&A, 413, L27

Tripathi, D., Isobe, H., \& Jain, R. 2009, SSRv, 149, 283

van Doorsselaere, T., Verwichte, E., \& Terradas, J. 2009, SSRv, 149, 299

Verwichte, E., van Doorsselaere, T., Foullon, C., \& White, R. S. 2013, ApJ, 767, 16

Walker, A. D. M. 1981, P\&SS, 29, 1119

Wright, A. N. 1992, JGR, 97, 6439

Wright, A. N., \& Rickard, G. J. 1995, ApJ, 444, 458

Xia, C., Keppens, R., Antolin, P., \& Porth, O. 2014a, ApJL, 792, L38

Xia, C., Keppens, R., \& Guo, Y. 2014b, ApJ, 780, 130 